\title{
Implantación de sistemas de información empresarial
}

\author{
Por Sabin Goitia, Sonia Sáenz-de-Lacuesta y Maitane Bilbao
}

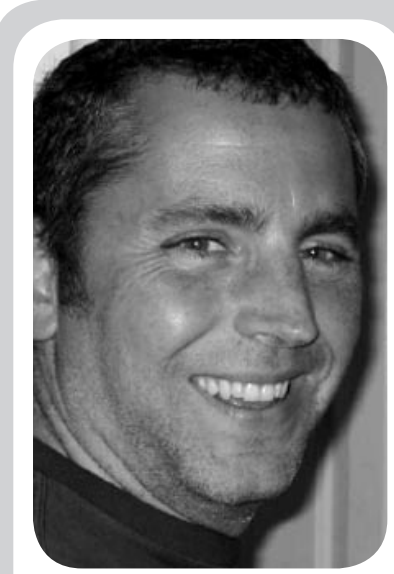

Sabin Goitia es ingeniero industrial especialidad Organización Industrial por la Escuela de Ingenieros Industriales y de Telecomunicaciones de Bilbao (UPV/EHU). Desde hace 6 años es el responsable de producto del servicio Zaintek.

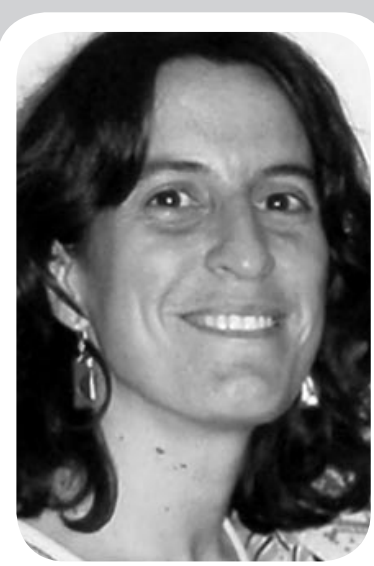

Sonia Sáenz de Lacuesta es licenciada en documentación por la Universitat Oberta de Catalunya (UOC) y desde hace 7 años es la gestora de información del servicio Zaintek.

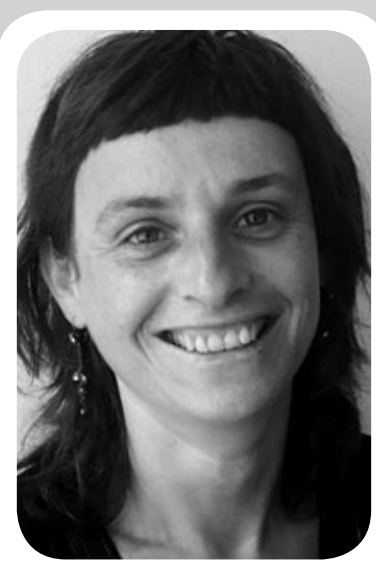

Maitane Bilbao es diploma da en secretariado de dirección y desde hace 7 años es técnico de vigilancia y estrategia en gestión de personal.
Resumen: En 2002 el Departamento de Innovación y Promoción Económica de la Diputación Foral de Bizkaia creaba el Servicio de Vigilancia Estratégica Competitiva (Zaintek) con el objetivo de sensibilizar a las pymes de Bizkaia sobre el uso de la información como herramienta de gestión estratégica. Para lograrlo puso en marcha a través de su web una serie de servicios de apoyo que han ido ganando en complejidad. Uno de ellos es el Sistema de Información Empresarial (SIE) a la medida de las pymes, que describiremos en este artículo.

En 2005 Zaintek se integró en la Agencia de Innovación de Bizkaia (BAI), hecho demostrativo de la importancia que la administración pública concede a la Vigilancia como medio de apoyo a la innovación.

Palabras clave: Información estratégica, Sistemas de información empresarial (SIE), Vigilancia tecnológica, Inteligencia competitiva, Pymes

\section{Title: Business information systems in companies}

Abstract: In 2002 the Department of Innovation and Economic Promotion of the Provincial Council of Bizkaia set up the Zaintek Strategic Competitive Surveillance service to increase awareness among SMEs in Bizkaia concerning the use of information as a tool for strategic management. To that purpose, Zaintek has focused on awareness and on developing a number of increasingly complex support services via its website. One of the most highly-developed services is a business information system (BIS) tailored to SMEs. This article examines the prerequisites for the implementation of this service, its objectives and its stages of development. Three years ago Zaintek was integrated into BAI (the Bizkaia Innovation Agency), evidencing the importance attributed by the public administration to surveillance as a support tool at all levels of innovation.

Keywords: Strategic information, Business information systems (BIS), Technology surveillance, Competitive intelligence, SMEs

Goitia, Sabin; Sáenz-de-Lacuesta, Sonia; Bilbao, Maitane. Implantación de sistemas de información empresarial. En: El profesional de la información, 2008, v. 17, n. 5, pp. 540-545.

DOI: 10.3145/epi.2008.sep.08

\section{La información estratégica en la empresa}

POCAS O NINGUNA ORGANIZACIÓN ACTUAL puede plantearse su participación competitiva en el mercado sin hacer esfuerzos por desarrollar una rápida y eficaz capacidad de adaptación.

Hay circunstancias, como la globalización de los mercados, la evolución vertiginosa de las tecnologías, o el nuevo papel -mucho más "sabio" y activo- que han asumido los consumidores, que afectan a cualquier empresa, independientemente de su escala.

En este contexto, lo único que permanece es el cambio. De ahí la importancia de buscar la forma de que las inherentes imprecisiones que se producen no se traduzcan en dificultades duras de afrontar, sino en algo que puede llegar a ser, incluso, estimulante y adecuado para mejorar los resultados. El cambio es igual para todos por lo que se trata de adaptarse a las nuevas situaciones mejor y antes que los competidores. No es casualidad que conceptos como flexibilidad, trabajo en red, o producción a la carta, se estén extendiendo en las organizaciones. Tampoco lo es que la información estratégica esté cobrando cada día 
mayor importancia, pues sin ella es imposible el análisis y la reflexión que exige la dinámica empresarial actual.

Las grandes corporaciones son las que apuestan con mayor decisión por la inteligencia competitiva. Es algo que les permite identificar anticipadamente oportunidades de mejora, prever tendencias, $\mathrm{y}$, sobre todo, pensar en futuro.

Sin embargo, la valoración que las pequeñas y medianas empresas hacen de la información estratégica sigue siendo relativamente baja. Es probable que si les preguntásemos si la consideran interesante para su futuro, la mayoría contestaría que sí, pero la realidad demuestra que el acercamiento sigue siendo esporádico.

Inmersas en el día a día, la incorporación de nuevas tareas es de por sí siempre difícil; la información tiene además unas características (volumen, veracidad, interés, disponibilidad, actualización constante, etc.) que hacen que su uso sólo sea válido si se elige y se utiliza de tal manera que responda bien a las necesidades precisas de cada empresa.

\section{Apoyo institucional a las pymes de Bizkaia}

Con el fin de acercar esta disciplina a las pymes de Bizkaia, el $D e$ partamento de Innovación y Promoción Económica de la Diputación Foral de Bizkaia creó en 2002 el Servicio de Vigilancia Estratégica Competitiva (Zaintek), que en su momento fue pionero. Es cierto que había empresas que ofrecían servicios de vigilancia sectorial, pero no instituciones públicas que pusiesen al alcance de las pymes un servicio permanente de vigilancia, respaldado por ayudas económicas.

Entre otros servicios, Zaintek desarrolla continuamente iniciativas de sensibilización sobre el uso de la información como herramien- ta de gestión estratégica y mantiene un programa de formación que enseña a las empresas a vigilar por sí mismas.

\section{http://www.zaintek.net}

El servicio más demandado es el de consultas para resolver cuestiones puntuales y expertas sobre proveedores, distribuidores, clientes potenciales, situación económica-financiera de un competidor, nuevas reglamentaciones, aparición de nuevas tecnología y/o procesos productivos, análisis comparados de patentes, etc.

En 2005, el mismo departamento foral que había puesto en marcha Zaintek decidió crear la Agencia de Innovación de Bizkaia (BAI) e integrar en ella Zaintek. Ratificaba así su interés por la información estratégica y su convencimiento de que ésta es un recurso competitivo clave para la innovación.

\section{http://www.bai.bizkaia.net}

A medida que el Servicio alcanzaba madurez, también lo han hecho las herramientas que oferta, hasta llegar a la elaboración de una metodología para implantar un Sistema de Información Empresarial a la medida de las pymes.

\section{Sistemas de Información Empresarial}

Un SIE es un conjunto estructurado de elementos que sirve para la captación, análisis, tratamiento, difusión y utilización sistemática de la información y el conocimiento necesarios para la correcta toma de decisiones en la empresa. Es, por tanto, un sistema de inteligencia de negocio.

La inteligencia de negocio hace referencia a las aplicaciones y técnicas que se usan para recopilar, acceder y analizar datos e información sobre las operaciones de la empresa, con objeto de disponer de conocimiento completo y exhaustivo, en tiempo y calidad, de los fac- tores internos y externos que afectan al negocio, ayudando a tomar decisiones más sustentadas.

Por otra parte, dichas aplicaciones facilitan la comunicación entre departamentos y procesos, coordinan actividades y permiten a las empresas responder de manera más rápida a los cambios.

\section{Claves de un SIE}

Como ya se ha dicho, un SIE debe adaptarse a las necesidades concretas de cada organización y a su estructura organizativa. Cuando se piensa en una instalación nunca se parte de cero pues todas las empresas disponen de algún tipo de sistema de información, más o menos rudimentario, con distintos grados de calidad/fiabilidad y con niveles de accesibilidad mayores o menores, etc. Esa información debe contemplarse como parte del SIE.

Por otro lado, el método que propone BAI para instalar SIEs en las empresas marca unas pautas, pero buena parte de su eficacia radica en aspectos que no se concretan en ningún manual y que ponen la pelota en el campo de la empresa.

Desde nuestra experiencia sabemos que para implantar con éxito un SIE se tiene que cumplir una serie de condiciones previas:

- considerar que es una actividad integrada en el resto de actividades de la empresa;

- implicación total de la Dirección de la empresa no sólo a la hora de implantar el sistema, sino también en su gestión diaria y, por supuesto, en la explotación de sus resultados en la estrategia empresarial;

- como toda actividad, necesita unos recursos mínimos;

- nombramiento de un responsable o animador-coordinador; y es recomendable también que esta función se realice a través de una unidad específica que se sitúe cerca de la Dirección; 
- clima favorable a la comunicación y al trabajo en equipo que permita compartir información y conocimiento entre los diferentes departamentos.

\section{EI SIE en tres fases}

La puesta en marcha de un SIE se articula en tres fases sucesivas: Prediagnóstico, Diagnóstico e Implantación.

En la figura se puede visualizar el proceso completo.

Para sintetizar la metodología de manera clara y concisa, hemos optado por explicar resumidamente en qué consiste cada fase, cuáles son sus objetivos, los riesgos que conlleva, así como su duración estimada.

\section{Fase 1: Prediagnóstico}

Es una etapa de recopilación de información, pensada para que los consultores que van a apoyar la implantación del SIE conozcan con detalle la empresa y para que ésta entienda bien qué es un SIE, qué beneficios aporta y cuáles son las implicaciones que su desarrollo va a tener en toda la organización.

Sus principales objetivos son:

- recabar información general de la empresa;

- valorar la conveniencia e interés de realizar un Diagnóstico;

- visualizar las necesidades adicionales de información;

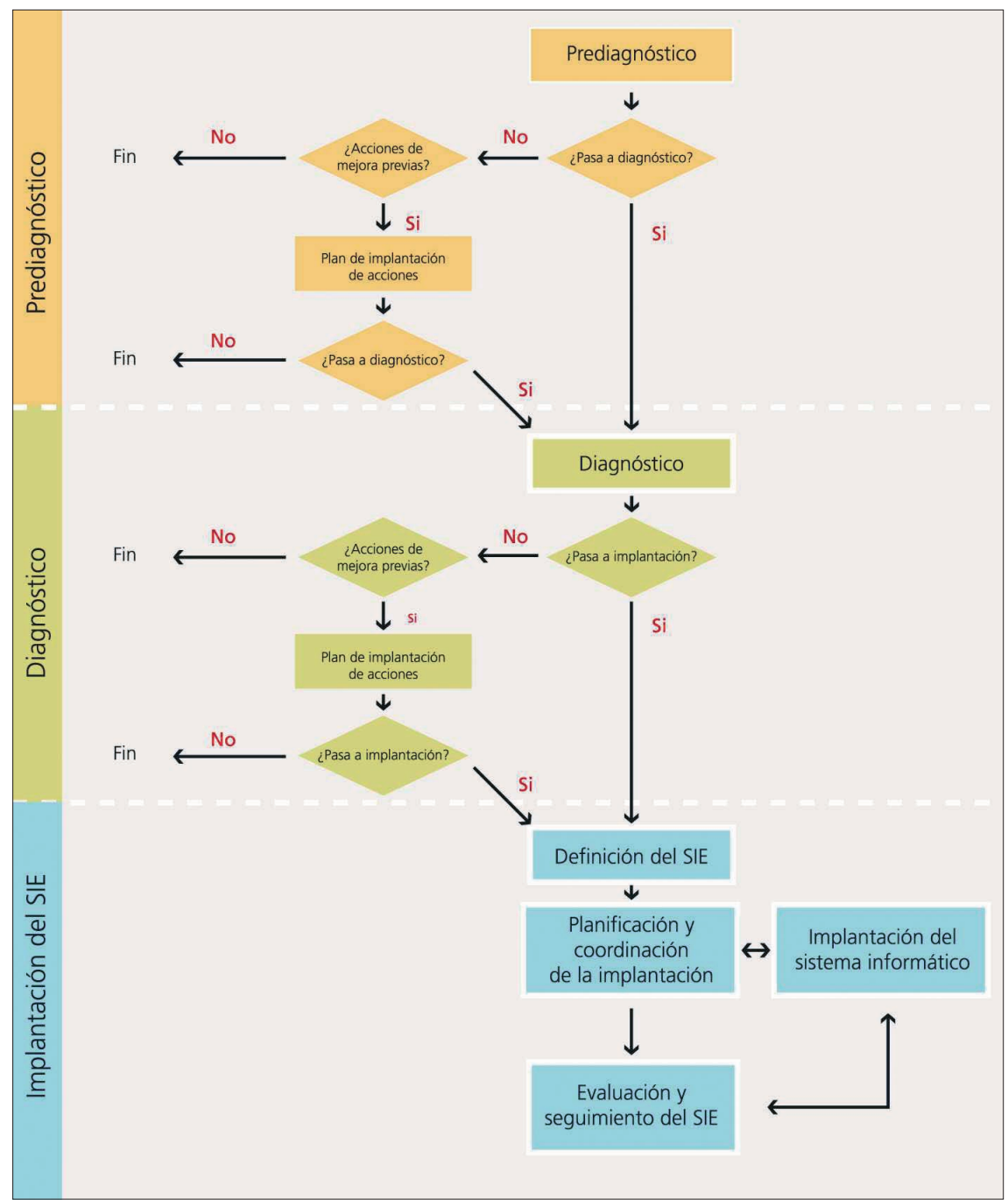

Esquema general de la implantación de un SIE

- reflexionar sobre las posibles acciones de mejora que pueden aplicarse al sistema de información del que ya disponen;

- obtener la información necesaria para planificar el Diagnóstico (alcance, plazo, personas a entrevistar, equipo de proyecto, recursos disponibles para el proyecto, metodología, presupuesto.

Hemos observado que dos son los riesgos más frecuentes que se dan en esta etapa (Cuadro 1):

Se estima que el tiempo necesario para elaborar un Prediagnóstico es de dos semanas, aunque depen-

\begin{tabular}{|l|l|}
\hline \multicolumn{1}{|c|}{ Riesgo } & \multicolumn{1}{c|}{ Recomendaciones } \\
\hline $\begin{array}{l}\text { La Dirección no es consciente de lo } \\
\text { que quiere o no lo ha explicado }\end{array}$ & $\begin{array}{l}\text { - Aclarar con el consultor el origen de la solicitud (es una demanda de } \\
\text { la empresa, se ha detectado en una visita comercial...). } \\
\text { - Explicar previamente a la reunión inicial el objetivo de la misma, } \\
\text { la duración, y hacer hincapié en la utilidad del Prediagnóstico en } \\
\text { particular y del SIE en general. } \\
\text { - Exponer lo que es un SIE, lo que aporta a la empresa y lo que les va } \\
\text { a exigir. }\end{array}$ \\
\hline $\begin{array}{l}\text { Emplear demasiado tiempo en la } \\
\text { realización del Prediagnóstico }\end{array}$ & $\begin{array}{c}\text { - En el Prediagnóstico ceñirse a la información necesaria para analizar } \\
\text { la conveniencia de seguir con el Diagnóstico. } \\
\text { - No volver a analizar en el Diagnóstico las cuestiones que ya se han } \\
\text { revisado en el Prediagnóstico. }\end{array}$ \\
\hline
\end{tabular}


derá en gran medida de la disponibilidad de la dirección de la empresa y la consultora para fijar las reuniones.

A partir de los resultados obtenidos, se evaluará si la compañía está en condiciones de abordar la siguiente etapa o si es más interesante definir un plan de acciones de mejora que permita luego a la empresa pasar con mayores garantías a la fase de Diagnóstico.

\section{Fase 2: Diagnóstico}

En esta fase se trata de hacer una radiografía del Sistema de Información que ya tiene la organización. Se trata, como decíamos anteriormente, de aprovechar esos recursos ya disponibles y tenerlos en cuenta para determinar el punto de partida y la estructura de su SIE específico.

Los objetivos de esta etapa son:

- detectar las informaciones usadas en la empresa;

- identificar aquellas que requieren una mejora sustancial;

- visualizar las necesidades adicionales de información;

- cualificar los flujos de información existentes: elaboradores de informaciones, utilizadores, uso...;

- predisponer positivamente a las distintas figuras / cargos en relación con el SIE:

- identificar las áreas clave de vigilancia;

- obtener conclusiones respecto a la factibilidad de abordar un proceso de implantación de un SIE.

En el Cuadro 2 se citan algunos de los riesgos más habituales que suelen darse en esta fase y se hacen algunas recomendaciones que pueden eliminarlos o minimizarlos.

Se estima que el tiempo necesario para cumplimentar esta fase puede llegar a ser de ocho semanas de trabajo, aunque dependerá de la complejidad de la compañía, del estado inicial de la información existente, de los posibles "parones" para profundizar en un área concreta, y de la disponibilidad del equipo de Diagnóstico.

Al igual que en la etapa anterior, una vez concluida se hará una valoración y se estimará si la empresa

\begin{tabular}{|c|c|}
\hline Riesgo & Recomendaciones \\
\hline $\begin{array}{l}\text { Que la Dirección general no asuma } \\
\text { el liderazgo interno del proyecto. }\end{array}$ & $\begin{array}{l}\text { - Es necesario dejar claro desde el primer momento que el SIE no ata- } \\
\text { ñe a uno o varios departamentos, sino que afecta o puede afectar a } \\
\text { toda la empresa. } \\
\text { - Puede ser un instrumento muy potente para mejorar el nivel de } \\
\text { gestión de la empresa y su proceso de toma de decisiones. Por ello } \\
\text { es imprescindible el liderazgo de la Dirección general, si no es mejor } \\
\text { no abordar el proyecto. }\end{array}$ \\
\hline $\begin{array}{l}\text { Falta de dedicación o de } \\
\text { atención suficiente por parte } \\
\text { de los miembros del equipo de } \\
\text { Diagnóstico. }\end{array}$ & $\begin{array}{l}\text { - Invertir recursos en que las personas de la empresa entiendan cla- } \\
\text { ramente lo que se espera de ellas, que no tengan dudas de lo que } \\
\text { tienen que preparar. Para ello es importante facilitarles algún ejem- } \\
\text { plo de lo que se va buscando y un medio de contacto para resolución } \\
\text { de dudas. } \\
\text { - Dejar claro desde el primer momento que la reunión del equipo de } \\
\text { Diagnóstico no se celebrará hasta que el consultor disponga de la } \\
\text { información adecuada. } \\
\text { - Transmitir sin demora este tipo de situaciones al coordinador inter- } \\
\text { no. }\end{array}$ \\
\hline $\begin{array}{l}\text { A la hora de identificar } \\
\text { informaciones bajar hasta un nivel } \\
\text { de detalle excesivo. }\end{array}$ & $\begin{array}{l}\text { - La empresa maneja cantidades ingentes de información. Hay que } \\
\text { dejar claro que debemos centrarnos en aquella que consideramos } \\
\text { clave para el desarrollo habitual de la actividad y que afecta a la } \\
\text { toma de decisiones operativas y/o estratégicas. }\end{array}$ \\
\hline $\begin{array}{l}\text { Monopolización de las reuniones } \\
\text { del equipo de Diagnóstico por una } \\
\text { o varias personas. }\end{array}$ & $\begin{array}{l}\text { - Fomentar la participación de todas las personas del equipo, incenti- } \\
\text { vando a las menos participativas con preguntas directas. } \\
\text { - No conviene olvidar que el Diagnóstico debe ser consensuado por } \\
\text { todos los miembros del equipo ya que el SIE afecta a toda la empre- } \\
\text { sa. }\end{array}$ \\
\hline $\begin{array}{l}\text { Realizar un análisis exhaustivo } \\
\text { y en profundidad de todo el } \\
\text { conjunto de la empresa. }\end{array}$ & $\begin{array}{l}\text { - Transmitir que debemos optimizar los recursos y por lo tanto hay } \\
\text { que centrarse en lo importante. } \\
\text { - Debemos generar un análisis lo suficientemente completo pero asu- } \\
\text { mible para los recursos de la empresa. }\end{array}$ \\
\hline $\begin{array}{l}\text { Elaboración de documentación } \\
\text { voluminosa. }\end{array}$ & $\begin{array}{l}\text { - Tratar de dar un enfoque esquemático a los documentos, evitando } \\
\text { en lo posible los grandes desarrollos explicativos. }\end{array}$ \\
\hline
\end{tabular}


está en condiciones de abordar un proyecto de implantación de un SIE o si es preciso realizar mejoras previas.

\section{Fase 3: Implantación del SIE}

Teniendo en cuenta los objetivos estratégicos de la empresa, se determina qué información/conocimientos le ayudarían a soportarlos. A partir de ahí, valorando la información disponible y la no disponible pero necesaria, se define el SIE y se pasa a su implantación y la del sistema informático de apoyo que va a permitir gestionarlo.

Para ello se llevan a cabo las siguientes acciones:

- Identificar los retos estratégicos, priorizando los tres principales, las decisiones unidas a esos retos y la información necesaria para la toma de decisión. Se detectan posibles riesgos, factores de éxito y se propone una forma de seguimiento.

- Desplegar los retos a los distintos departamentos de la empresa, determinando las decisiones a tomar en cada uno de ellos y la información que soporte dichas decisiones.

- Comparar la información anterior con la identificada para cada proceso en la etapa de Diagnóstico, seleccionando y priorizando la información a incorporar al SIE.

- Documentar la información indicando las fuentes, los responsables de su captación, tratamiento y uso, y la periodicidad.

- Organizar la información de acuerdo con la estructura del SIE: área de vigilancia-área temática-Información.

- Definir la organización de soporte.

- Planificar los aspectos relacionados con la implantación del SIE, recapitulando sobre posibles acciones pendientes.

- Analizar, planificar e implantar el sistema informático de apoyo que sirva para gestionar el SIE, el más adecuado para seleccionar, extraer, analizar y difundir la información.

- Realizar las acciones necesarias para la instalación del SIE y del soporte informático.

- Estructurar y llevar a cabo la evaluación y seguimiento del grado de operatividad del SIE y de su eficiencia en función de los indicadores elegidos.

La implantación de un SIE en una pyme tiene una duración estimada de 6 meses. La duración real, sin embargo, puede variar en función de la mayor o menor complejidad del área de actividad de la empresa, de la situación de su sistema de información previo, y del tiempo que precise el desarrollo de la plataforma tecnológica (en caso de que no se utilice la herramienta de gestión propuesta por Zaintek).

\section{Conclusiones}

La información estratégica en las empresas

El conocimiento es el motor de las organizaciones actuales. La empresa siempre ha considerado importante la información y ha tratado de interpretarla con visión de futuro, pero la globalización y el desarrollo de la Red digital la han convertido en un recurso estratégico clave y ha hecho que las fuentes sean ilimitadas.

Eso ha hecho que disciplinas como la inteligencia competitiva, dedicada a recoger, filtrar $\mathrm{y}$ analizar la información, sean hoy un elemento esencial para cualquier organización.

\section{El apoyo institucional a las py- mes}

Las pymes esperan apoyo de las instituciones, económico por supuesto, pero también que les "empujen" a abordar nuevos proyectos, a buscar nuevos aliados, a utilizar en su beneficio las nuevas disciplinas que surgen.

La experiencia nos dice que no hay pyme que no tenga ideas nuevas regularmente o que no intuya qué recursos necesitaría para ser competitiva, pero la mayoría de las veces los pensamientos se quedan ahí: ni se encuentra el momento ni se conocen los canales para realizarlos.

Entendemos que la Administración debe asumir ese papel dinamizador y poner en marcha servicios que, en este caso concreto, ayuden a concienciar, entender y a gestionar la información estratégica.

\section{Concienciación empresarial}

Iniciábamos este texto hablando de flexibilidad, la metodología Sistema de Información Estratégica de BAI también la tiene. Sugiere un camino que considera clave tener en cuenta las circunstancias particulares de cada organización en la que se implante y adaptar la herramienta a su idiosincrasia.

Lo verdaderamente interesante es que la empresa asuma una dinámica de vigilancia que le lleve a ir profundizando en los mecanismos de captura y análisis de la información. El SIE se irá perfeccionando y ganará madurez a medida que la organización vea los beneficios que reporta: mayor conocimiento, más seguridad en sus decisiones y mayor certeza en éstas, mayor margen de maniobra, más posibilidades de diferenciarse, mejores resultados, etc.

\section{Resumen de requisitos}

Otra idea importante es que las garantías de éxito de un proyecto de implantación del SIE son mayores cuando se parte con las ideas claras, de ahí que no queramos terminar estas líneas sin recordar las reglas básicas del juego:

- Hay que identificar con claridad las áreas de vigilancia de la empresa y asegurarse de su alineación con sus retos estratégicos.

- Es preciso adecuar los obje- 
tivos del SIE a los medios disponibles en la organización; objetivos ambiciosos pero realizables.

- Todas las personas de la empresa han de tener acceso al SIE y han de involucrarse en su mantenimiento.

- Los recursos de la empresa son limitados. Para lograr la eficiencia en su utilización, la organización debe apoyarse en todos los agentes del entorno disponibles cara a alimentar su SIE.

- Se debe dotar a la empresa de una plataforma tecnológica que permita agilizar el mantenimiento y la accesibilidad del SIE.
- El SIE es patrimonio de toda la empresa por lo que es imprescindible consensuar el proyecto.

\section{Bibliografía}

Zaintek, Modelos de vigilancia tecnológica e inteligencia competitiva, BAI Agencia de Innovación de Bizkaia, 2007

Zaintek, Vigilar para innovar, BAI Agencia de Innovación de Bizkaia, 2006.

Zaintek, El reto de los Sistemas de Información empresarial (SIE) en las empresas: acciones en 5 pymes de Bizkaia, BAI Agencia de Innovación de Bizkaia, 2005.

Zaintek, El reto de la Vigilancia Tecnológica en las empresas: acciones en 15 pymes de Bizkaia, BAI Agencia de Innovación de Bizkaia, 2005.

Zaintek, Manual de Implantación de un Sistema de Información Empresarial (SIE), DZ Centro de Diseño, 2004.
Zaintek, Guía de Vigilancia Tecnológica: Sistemas de Información estratégica en las pymes, DZ Centro de Diseño, 2003

Zaintek, Servicio de Vigilancia Estratégica Competitiva de BAI Agencia de Innovación de Bizkaia.

http://www.zaintek.net

CRIQ, Centro de Investigación Industrial de Québec.

http://www.criq.qc.ca

Sabin Goitia, Sonia Sáenz-deLacuesta y Maitane Bilbao. BAI Agencia de Innovación de Bizkaia, Servicio de Vigilancia Estratégica Competitiva (Zaintek). Sabino Arana, 8. 48013 Bilbao.

zaintek@bizkaia.net

http://www.zaintek.net

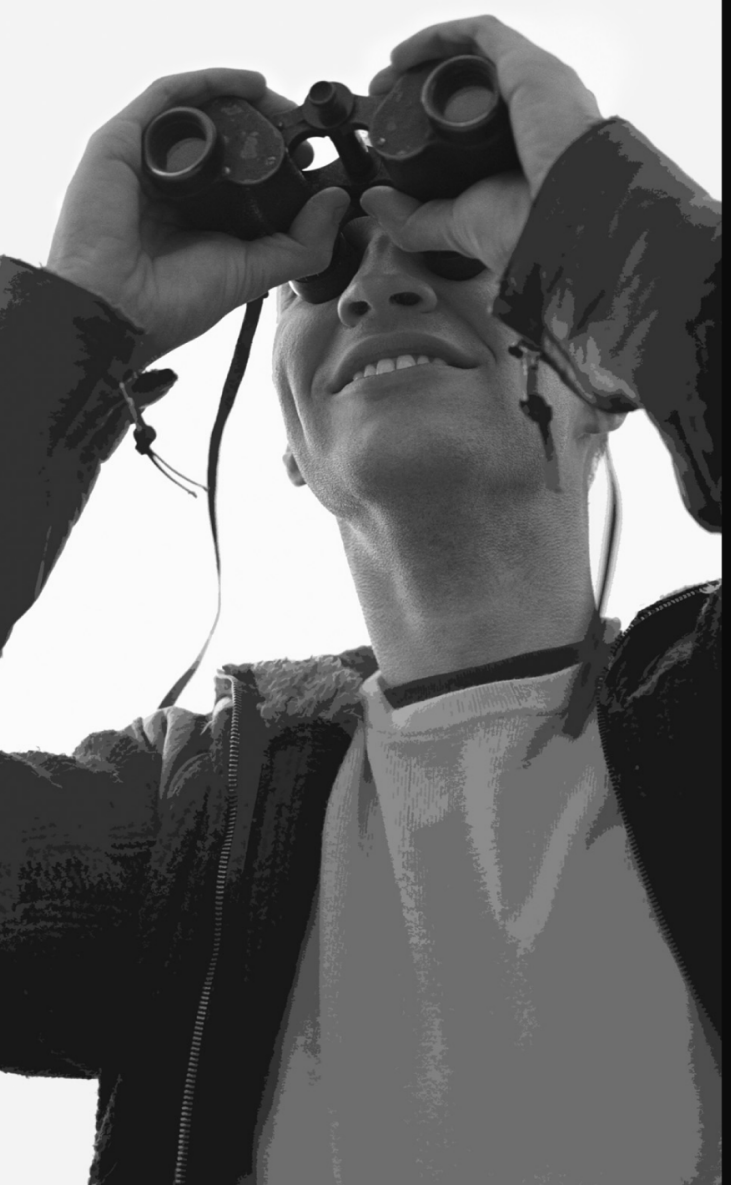

\section{MÁSTER EN DOCUMENTACIÓN DIGITAL}

Máster y Programas de posgrado online

Arquitectura de la información en sitios web. - Organización de la información y sistemas de gestión de base de datos. - Buscadores y posicionamiento web. - Documentación audiovisual. - Web 2.0 (nuevo). - Archivística digital. - Gestión de contenidos y gestión del conocimiento. - Repositorios y bibliotecas digitales (ampliado). - Usabilidad.

Dirección: Cristòfol Rovira y Lluís Codina

Calendario: del 9 de octubre de 2008 al 30 de junio de 2009.

www.documentaciondigital.org

\section{MÁSTER EN BUSCADORES MARKETING Y POSICIONAMIENTO}

Máster y Programas de posgrado online

- Fundamentos. - Posicionamiento en Buscadores. - Marketing en Buscadores. - Vigilancia Tecnológica. . Recuperación de la Información. • Buscadores especializados.

Dirección: Cristòfol Rovira y Lluís Codina

Calendario: del 13 de noviembre de 2008 al 30 de junio de 2009.

www.masterenbusctadores.com

\section{Colaboran:}

Observatorio de Comunicación Científica - UPF Instituto Universitario de Lingǘstica Aplicada - UPF DOC 6 Consultores de Recursos de Información 